\title{
Performance Enhancement of MC-CDMA System through STBC based STTC Site Diversity
}

\author{
N. Kumaratharan, S. Jayapriya and P.Dananjayan
}

\begin{abstract}
The combination of multiple antennas and multi-carrier code division multiple access (MC-CDMA) is a strong candidate for the downlink of future mobile communications. The study of such systems, in scenarios that model real life transmissions is an additional step towards an optimised achievement. Nevertheless, when transmitting over fading channel multi-cell interference occurs and this degrades the performance of the system. Site diversity technique is applied to the system to overcome multi-cell interference. Due to non orthogonality of spreading codes multi-cell interference is not completely eradicated. To overcome this problem, spreading codes are assigned to each base station. Space time trellis code (STTC) site diversity with multiple input multiple output (MIMO) technique was introduced to reduce multi-cell interference further. In this paper balanced STTC (B-STTC) site diversity is proposed to improve the performance of MC-CDMA system and is extended to space time block code (STBC) based STTC site diversity. Simulation result shows that STBC based STTC site diversity outperforms B-STTC site diversity.
\end{abstract}

Index Terms-B-STTC, MC-CDMA, STBC based STTC, STTC.

\section{INTRODUCTION}

Broadband wireless access for evolving mobile internet and multimedia services are driving a surge of research on future wireless communication systems to support multi-user access and high data rates. Multi-carrier code division multiple access (MC-CDMA), which suits high data rate applications with multiplexing technique appears to be a promising technique in achieving high data rates [1]. MC-CDMA is robust to multi-path fading, inheriting the advantages of conventional CDMA where frequency diversity can be achieved in a broadband channel [2]. With its capability of synchronous transmission, MC-CDMA is suitable for downlink of cellular communication systems [3]. The challenge of achieving reliable data transmission over wireless link is more difficult due to the fact that received signals from multi-paths add destructively causing multi-cell interference which results in serious performance degradation. To achieve reliable communication over wireless links antenna diversity [4] derived by employing spatially separated antennas at the transmitter and receiver

Corresponding author is with the Department of Electronics and Communication Engineering, Pondicherry Engineering College, Pondicherry, India. (e-mail: pdananjayan@rediffmail.com) was introduced. High data rate MC-CDMA systems additionally employs multiple input multiple output (MIMO) techniques to mitigate fading [5].

Data transmission involves spreading operations by the use of short channelisation code and long scrambling code. Short channelisation code helps in separating the signals of different users present within the cell and long scrambling code mitigates the effects of interference produced by users belonging to other cells. However, the system faces multi-cell interference due to fading channel resulting in degradation of bit-error rate (BER).

Site diversity technique has been proposed for realizing CDMA and orthogonal frequency division multiplexing (OFDM) systems to minimize multi-cell interference [5-7], where space time block code (STBC) is used to gain diversity effect among several base stations. STBC site diversity system transmits the encoded signals from several base stations and these signals are combined at the receiver with STBC decoding operation. STBC branches and the scrambling codes are assigned to each base station to maintain orthogonality of signals between the cells and to reduce interference among them. The same technique is extended to MC-CDMA system. However, the scrambling codes assigned are generally non orthogonal among cells and hence multi-cell interference still exists. Using STBC with multiple antennas at each base station, site diversity was achieved with further reduction in multi-cell interference [8]. STBC does not provide coding gain and in view of this it is worthwhile to consider a joint design of error control coding, modulation, transmit and receive diversity to develop an effective signalling scheme called space time trellis code (STTC) [9], which combats the effects of fading. STTC became extremely popular as it can simultaneously offer coding gain with spectral efficiency and full diversity over fading channels. STTC was used to obtain site diversity with multiple antennas at base station and it outperformed STBC based site diversity in terms of error rates [10]. A new class of 4- phase shift keying (4-PSK) STTC using points of constellation with same probability called as balanced STTC (B-STTC) [11] was proposed for multiple transmit antennas. STBC based STTC codes built with set partitioning introduced in [12], achieves more diversity gain and achieves better performance in terms of error rates. In this work B-STTC with multiple antennas at base station is used to obtain site diversity for MC-CDMA system. Further, it is 
extended to STBC based STTC to obtain site diversity.

\section{StTC Site Diversity TeChNiqUe FOR MC-CDMA SySTEM}

The system model of space-time trellis coded modulation with $N$ transmit antennas and $M$ receive antennas are shown in Fig. 1 and 2 respectively. The information symbol $s(l)$ at time $l$ is encoded by the space-time encoder as $N$ complex base-band symbols. Each base-band symbol $c_{i}(l)$ is transmitted simultaneously from different antenna. A space-time code symbol $c(l)$ is an $N$ column vector $c(l)=\left[c_{1}(l), c_{2}(l), \ldots, c_{N}(l)\right]$. The signal obtained at the receive antenna is superposition of the faded version of the signals from $N$ transmit antennas. The received signal at antenna $j$ is given as

$$
r_{j}(l)=\sqrt{E_{s}} h_{i j}(l) c_{i}(l)+n_{j}(l), i=1, \ldots, N ; j=1, \ldots, M
$$

where $E_{s}$ is the average energy of the signal constellation

$$
h_{i j}(l) \text { is the path gain at time } l
$$

$n_{j}(l)$ is the noise present at the receive antenna at time $l$.

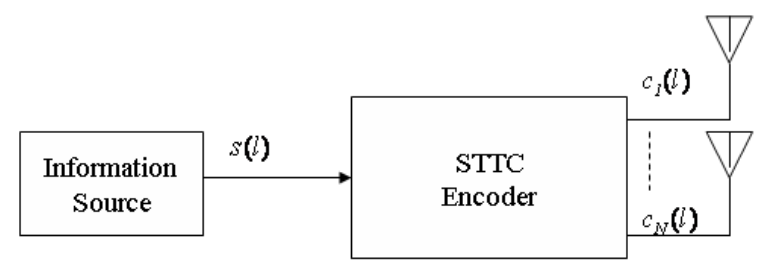

Figure 1. Block diagram of STTC transmitter

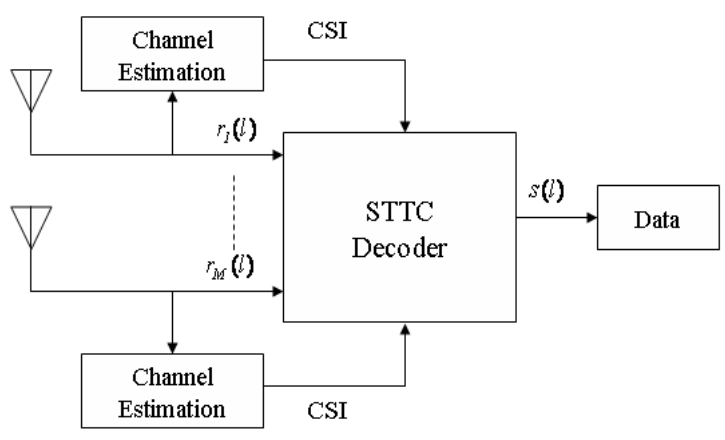

Figure 2. Block diagram of STTC receiver

The space-time decoder needs to know the path gains, also called channel state information (CSI), to decode the transmitted codeword. The received signal $r_{i}(l)$ is weighted by the path gains and the Euclidean distance is used as the input to the maximum likelihood vector detector for decoding process.

STTC often described by the trellis diagram as illustrated in Fig. 3 or the encoder structure for generator matrix as given in Fig.4 for 4-state STTC with two-transmit antennas. The trellis diagram is similar to those used in the trellis coded modulation (TCM). State bits are shown at the right of the trellis; each line represents a possible transition with the input bits shown besides the line. Current state outputs and inputs are shown in the matrix at the left of the trellis and are grouped together for different transmit antennas. Symbol bits are fed as input to the upper and lower branches. The branch coefficients are arranged alternatively in the generator matrix, with $a_{i}$ representing the most significant bit and $b_{i}$, the least significant bit. The output of the encoder is computed as

$x_{t}^{k}=\left(\sum_{p=0}^{v 1} I_{t-p}^{1} a_{t-p}^{k}+\sum_{q=0}^{v 2} I_{t-p}^{2} b_{t-q}^{k}\right) \bmod 4, k=1,2$

where $I$ is the input symbol bits to the generator matrix $v 1$ is the memory order of the upper branch $v 2$ is the memory order of the lower branch.

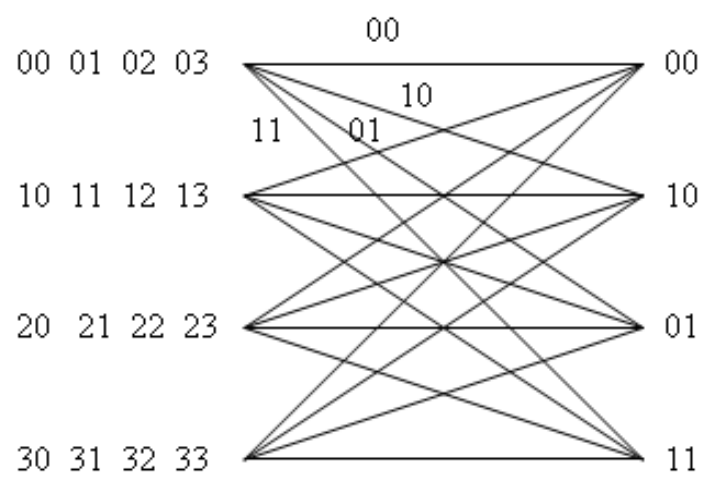

Figure 3. Trellis diagram for STTC

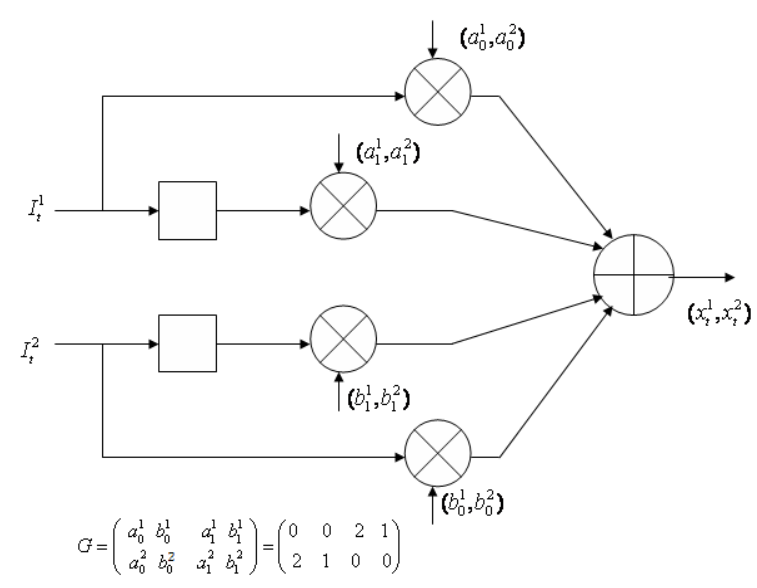

Figure 4. Matrix generator for STTC

When a codeword $c=\left(c_{1}, c_{2}, \ldots, c_{l}\right)$ with length $l$ is transmitted the codeword length and the maximum likelihood receiver might decide erroneously in favour of another codeword $e=\left(e_{1}, e_{2}, \ldots, e_{l}\right)$. Matrix $B$ of size $N \times l$ was constructed with elements $\left(e_{j}^{i}-e_{j}^{i}\right), i=1, \ldots, N ; j=1, \ldots, l$ and $r$ denotes the rank of the matrix $B$ with eigen values $\lambda_{i}$, the distance matrix $A$ is calculated as $A=B \cdot B^{*}$. With this the performance based on rank and determinant criteria and Euclidean distance criteria of space-time codes over Rayleigh fading channels, assuming perfect CSI are made available to the receiver.

When diversity gain $r M$ is small, the rank and determinant criteria will determine the performance [13]. When the 
diversity gain is large (with more number of antennas), the Euclidean distance criterion will determine the performance of STTC in terms of BER [14].

\section{B-StTC Site Diversity TeChNiQue For MC-CDMA SYSTEM}

A new class of 4- phase shift keying (4-PSK) STTC using points of constellation with same probability called as B-STTC [11] is proposed for multiple transmit antennas. Compared to the known codes like STBC and STTC, B-STTC offers best performance in terms of error rates and therefore systematic search for good codes can be reduced to B-STTC.

Considering a binary input data generated by a memory less source $S=\{0,1\}$ with equally probable symbols and the modulation for a given state $X=\left[x_{1} x_{2}, \ldots, x_{n}\right]^{T}$ with shift register realized by $(v+1)$ blocks of $n$ bits. The MIMO symbol $Y=\left[\begin{array}{lll}y_{1} & y_{2}, \ldots, y_{n}\end{array}\right]^{T}$ generated by the STTC encoder is given by a deterministic relation

$$
Y=C \cdot X
$$

where $C$ is the generator matrix given by

$$
C=\left(\begin{array}{ccccccc}
c_{1,1}^{1} & \ldots & c_{n, 1}^{1} & \ldots & c_{1, v+1}^{1} & \ldots & c_{n, v+1}^{1} \\
c_{1,1}^{k} & \ldots & c_{n, 1}^{k} & \ldots & c_{1, v+1}^{k} & \ldots & c_{n, v+1}^{k} \\
c_{1,1}^{n T} & \ldots & c_{n, 1}^{n T} & \ldots & c_{1, v+1}^{n T} & \ldots & c_{n, v+1}^{n T}
\end{array}\right)
$$

Here STTC is defined by mapping, such that state $X$ associates to codeword $Y$ and can be obtained for several states.

Due to the random source $S=\{0,1\}$, for a given state $X$ the encoder can have only 4 equally probable next states and the transition probabilities between these states corresponds to a Markov chain. The steady state probabilities of the states $X$ are all equal as the matrix is symmetrical. For a balanced code, by using (3), the generated codeword $Y$ is also equally probable, or in other words the generated symbols of the constellation are with equal probability.

\section{Properties of Balanced STTC}

The design of the B-STTC is based on the following properties:

Theorem 1: If a MIMO code with a L-length shift-register is fully balanced then $\mathrm{L}>\mathrm{L}_{\min }$.

Theorem 2: For a balanced MIMO code with L-length shift-register with any additional column matrix $C_{i}$, the resulting MIMO code with $(L+1)$ length shift-register is also balanced.

Definition 1: The vectors $C_{1}, C_{2} \ldots, C_{L}$ are linearly independent if

$$
\mathrm{x}_{1} \mathrm{C}_{1}+\mathrm{x}_{2} \mathrm{C}_{2}+\ldots .+\mathrm{x}_{\mathrm{L}} \mathrm{C}_{\mathrm{L}}=0
$$

with $\mathrm{x}_{\mathrm{i}} \in\{0,1\}$ holds if and only if all $\mathrm{x}_{\mathrm{i}}=0$

Definition 2: A set of linearly independent vectors $C_{1}, \ldots, C_{m}$ is called a base, if and only if $\operatorname{span}\left(c_{1}, c_{2}, \ldots, c_{m}\right)=\left\{\sum_{i=1}^{m} x_{i} c_{i} / x_{i} \in\{0,1\}\right\}$

Balanced codes are generated based on the properties and it gives better performance compared to STTC. B-STTC is said to be fully balanced if and only if the generated codeword has the same number of occurrences. Using this B-STTC, site diversity is obtained for MC-CDMA system.

\section{Stbc Based StTc Site Diversity Technique For MC-CDMA SYSTEM}

STBC based STTC built by set partitioning [12] combines the coding advantage of STTC together with diversity advantage of STBC which outperforms STTC codes. The goal of set partitioning is to achieve a better coding gain and is achieved through pairwise distance. Depending on the kind of code the pairwise distance is defined, and for STBC, it is the determinant criterion and is used to establish partitioning rules. Set partitioning is based on minimum coding gain distance (CGD). If the transmission matrix of space time code is denoted as $c_{1}=G\left(x_{1}, x_{2}\right)$, where $x_{1}, x_{2}$ are the transmitted symbols and $D\left(c_{1}, c_{2}\right)=G\left(x_{1}, x_{2}\right)-G\left(x_{1}^{\prime}, x_{2}^{\prime}\right)$ represents the difference of the transmission matrices for codewords $c_{1}$ and $c_{2}$ then the diversity is defined by the minimum rank of the matrix $D\left(c_{1}, c_{2}\right)$. The minimum of the determinant of the matrix $A\left(c_{1}, c_{2}\right)=D^{H}\left(c_{1}, c_{2}\right) D\left(c_{1}, c_{2}\right)$ over all possible pairs of distinct codewords $c_{1}$ and $c_{2}$ corresponds to the coding gain. Using this definition CGD between codewords $\left(c_{1}, c_{2}\right)$ is given as

$C G D\left(c_{1}, c_{2}\right)=D^{2}\left(c_{1}, c_{2}\right)=\operatorname{det}\left(A\left(c_{1}, c_{2}\right)\right)$

where

$$
A\left(c_{1}, c_{2}\right)=\left[\begin{array}{ccc}
2\left|z_{1}\right|^{2} & -\left(z_{3}\right)\left(z_{1}\right)^{*} e^{j \beta} & \left(z_{3}\right)^{*}\left(z_{1}\right) \\
-\left(z_{3}\right)^{*}\left(z_{1}\right) e^{-j \beta} & \left|z_{1}\right|^{2}+\left|z_{3}\right|^{2} & \left(z_{3}\right)^{*}\left(z_{1}\right) e^{-j \alpha} \\
\left(z_{3}\right)\left(z_{1}\right)^{*} & \left(z_{3}\right)^{*}\left(z_{1}\right) e^{j \alpha} & 2\left|z_{3}\right|^{2}
\end{array}\right]
$$

$z_{1}=x_{1}-x_{1}^{\prime} ; z_{2}=x_{2}-x_{2}^{\prime} ; z_{3}=x_{3}-x_{3}^{\prime}$

The STBC orthogonal design coupled via symbol $x_{2}$ for two transmit and receive antenna is denoted as

$$
G\left(x_{1}, x_{2}, x_{3}\right)=\left[\begin{array}{ccc}
-x_{2}{ }^{*} & x_{1} & x_{1}{ }^{*} e^{j \beta} \\
x_{1}{ }^{*} & x_{2} & -x_{3}{ }^{*} \\
x_{3}{ }^{*} e^{j \alpha} & x_{3} & x_{2}{ }^{*}
\end{array}\right]
$$

where $x_{1}{ }^{*} e^{j \beta}$ and $x_{3}{ }^{*} e^{j \alpha}$ enable to optimise the minimum CGD within each obtained coset, angles $(\alpha, \beta)$ belong to the constellation symbol and are used to separate the triplets of symbols which share the same second symbol. 
The set portioning for 4-PSK constellation is obtained through the mathematical expression of

$\operatorname{det}\left(A\left(c_{1}, c_{2}\right)\right)=\left|z_{1}\right|^{4}\left|z_{3}\right|^{2}+\left|z_{1}\right|^{2}\left|z_{3}\right|^{4}-2 \operatorname{Re}\left[\left(z_{3}^{*}\right)^{3}\left(z_{1}\right)^{3} e^{j(\alpha-\beta)}\right]$

The choice of $(\alpha, \beta)$, which maximises the minimum CGD within each coset is done with a sampling rate of $\pi / 16$. However, the obtained values for $(\alpha, \beta)$ does not correspond to 4-PSK constellation points and this entails the need for constellation expansion to use set partitioning with maximum of minimum CGD values. Applying set partitioning for 16 cosets gives a minimum CGD values of 128 and without constellation expansion, using set partitioning for the same number of cosets gives minimum CGD of 8 with $(\alpha, \beta)=(0,0)$.

A design with full diversity is needed to use STBC code (8) into a STTC design and in fact, achieving full diversity is equivalent to showing the determinants of matrices $A\left(c_{1}, c_{2}\right)$ are nonzero over all possible codewords $c_{1}$ and $c_{2}$. As it is the case, matrices $A\left(c_{1}, c_{2}\right)$ should be checked that they do not loose the full rank vale when $c_{1}$ and $c_{2}$ belong to different cosets. To solve this unit transform matrices $\Phi$ are assigned to each state to check that the minimum of det ( $\left.A\left(c_{1}, c_{2}\right)\right)$ is equal to zero when $c_{1}$ belongs to coset 1 and $c_{2}$ belongs to coset 2 . in fact unitary matrix $\Phi_{j}$ corresponds to a rotation and preserves distance among the constellation points i.e., the minimum CGD value is left unchanged by applying a unitary transform $\Phi_{j}$ to $G\left(x_{1}, x_{2}, x_{3}\right)$ within each coset. Search for unitary matrices are done using parameterisation [15], and along with the selected partitioning level, unit matrices whose number equal to the number of cosets with maximum separation distance are obtained. The trellis is evenly built by affecting a unitary matrix transform to each coset with each of them corresponding to a trellis' state and is transmitted. The received signal is given as

$$
Y=H \Phi_{j} G\left(x_{1}, x_{2}, x_{3}\right)+N
$$

where

$\mathrm{H}$ is the channel coefficient $\left[h_{1}^{j} h_{2}^{j} h_{3}^{j}\right]$

$\Phi_{j}$ is the $3 \times 3$ unitary matrix used in state $\mathrm{j}$

$\mathrm{N}$ is the vector of additive white Guassian noise with zero mean and variance $\sigma^{2}$.

For a three receive antenna, signal obtained is given as

$$
\begin{aligned}
& y_{1}=-h_{1}^{j} x_{2}^{*}+h_{2}^{j} x_{1}^{*}+h_{3}^{j} x_{3}^{*} e^{j \alpha}+n_{1} \\
& y_{2}=h_{1}^{j} x_{1}+h_{2}^{j} x_{2}+h_{3}^{j} x_{3}+n_{2} \\
& y_{1}=h_{1}^{j} x_{1}^{*} e^{j \beta}-h_{2}^{j} x_{3}^{*}+h_{3}^{j} x_{2}^{*}+n_{3}
\end{aligned}
$$

To compute branch metrics for different symbol triplets, auxiliary quantities are obtained as

$$
\begin{aligned}
& y_{1}^{\prime}=y_{1}-h_{3}^{j} x_{3}^{*} e^{j \alpha} \\
& y_{2}^{\prime}=y_{2}-h_{3}^{j} x_{3} \\
& y_{3}^{\prime}=y_{3}-h_{1}^{j} x_{1}^{*} e^{j \beta}
\end{aligned}
$$

With these (11) and (12) received signal is obtained by combining technique and is given as

$$
Z=M X+N^{\prime}
$$

where

$$
\begin{gathered}
Z=\left[\begin{array}{c}
y_{1}^{*} h_{2}^{j}+y_{2} h_{1}^{j *} \\
-y_{1}^{*} h_{1}^{j}+2 y_{2} h_{2}^{j *}+y_{3}^{*} h_{3}^{j} \\
-y_{3}^{*} h_{2}^{j}+y_{2} h_{3}^{j *}
\end{array}\right] \\
N^{\prime}=\left[\begin{array}{c}
n_{1}^{*} h_{2}^{j}+n_{2} h_{1}^{j *} \\
-n_{1}^{*} h_{1}^{j}+2 n_{2} h_{2}^{j *}+n_{3}^{*} h_{3}^{j} \\
-n_{3}^{*} h_{2}^{j}+n_{2} h_{3}^{j *}
\end{array}\right]
\end{gathered}
$$

$$
M=\left[\begin{array}{ccc}
\left|h_{1}^{j}\right|^{2}+\left|h_{2}^{j}\right|^{2} & 0 & h_{3}^{j} h_{1}^{j *}+h_{2}^{j} h_{3}^{j *} e^{-j \alpha} \\
h_{1}^{j} h_{2}^{j *}+h_{3}^{j} h_{1}^{j *} e^{-j \beta} & \left|h_{1}^{j}\right|^{2}+2\left|h_{2}^{j}\right|^{2}+\left|h_{3}^{j}\right|^{2} & h_{3}^{j} h_{2}^{j *}+h_{1}^{j} h_{3}^{j *} e^{-j \alpha} \\
h_{1}^{j} h_{3}^{j *}+h_{2}^{j} h_{1}^{j *} e^{-j \beta} & 0 & \left|h_{2}^{j}\right|^{2}+\left|h_{3}^{j}\right|^{2}
\end{array}\right]
$$

\section{Simulation Results}

The proposed site diversity for MC-CDMA system is simulated using MATLAB and the simulation parameters are given in Table 1. Fig.5 shows the BER performance with respect to energy per bits to the spectral noise density $\left(\mathrm{E}_{\mathrm{b}} / \mathrm{N}_{0}\right)$ of the system with and without diversity under Rayleigh fading channel. The diversity technique uses two antennas at the transmitter and receiver terminal. The result indicates that when diversity was used there is an improvement in BER performance of the system due to the exploitation of multiple antennas in the transmitter and receiver.

TABLE I

SIMULATION PARAMETERS

\begin{tabular}{|l|l|}
\hline \multicolumn{1}{|c|}{ Parameters } & \multicolumn{1}{c|}{ Values } \\
\hline Modulation & 4 PSK \\
\hline Symbol length & 64 \\
\hline No.of sub- carriers & 128 \\
\hline Channel estimation & Perfect estimation \\
\hline Channelisation code & Walsh-Hadamard code of length 63 \\
\hline Scrambling code & Random code of length 63 \\
\hline Channel & Rayleigh fading channel with AWGN floor \\
\hline
\end{tabular}

Performance of MC-CDMA system with STTC site diversity is evaluated by varying the transmitting and receiving antennas. Fig. 6 illustrates the performance of the system in terms of symbol error rate (SER) and signal to noise ratio (SNR) with STTC site diversity for two, three, four and five transmit and receive antennas. It is observed from the plots that the system with five transmit and receive 
antennas gives better performance in SER when compared to the system with two, three and four antennas. The improvement in SER for the larger number of antennas is due to the maximum utilisation of diversity. The maximum number of antennas used for simulation is restricted to five as further increase of it introduces hardware complexity and increases the cost of the system.

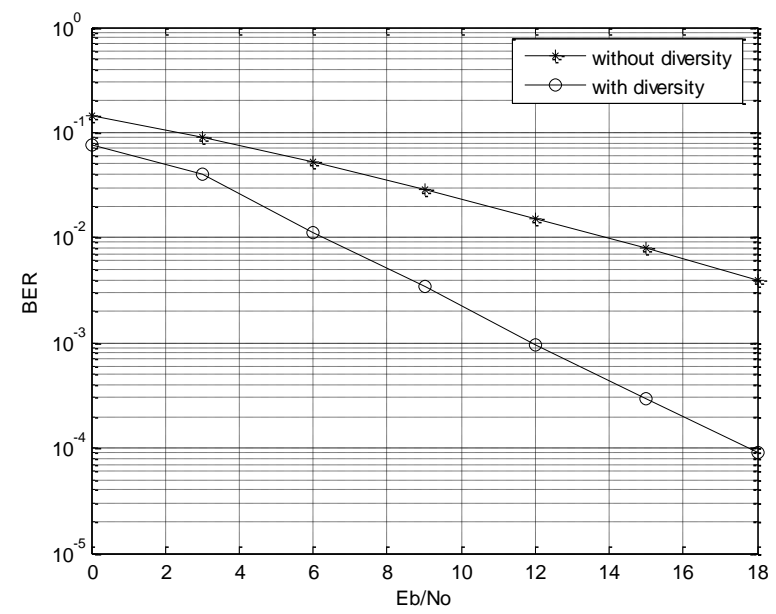

Figure 5. Performance of the system with and without diversity

Fig. 7 depicts SER versus SNR performance of the system with B-STTC site diversity for various numbers of antennas. The result portrays that the SER of the system with five transmit and receive antennas outperforms two, three and four transmit and receive antennas. Fig. 8 renders the same scenario of the system with STBC based STTC site diversity for different antennas. Similar conditions of diversity utilisation observed in Fig.6 and 7 are noticed here irrespective of the coding techniques used for site diversity i.e. improvement of SER is noticed clearly when there is increase in number of antennas.

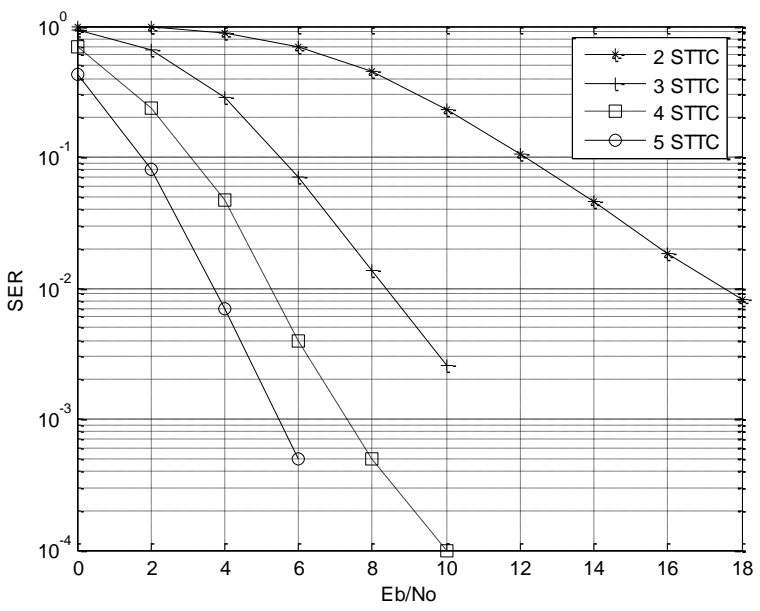

Figure 6. Performance of the system with STTC site diversity for

various antennas

SER performance is compared for STTC, B-STTC and STBC based STTC site diversity of the system for different transmit and receive antennas in Fig. 9. It is vivid that
B-STTC based site diversity outperforms STTC based site diversity as it achieves better coding rate compared to STTC since they use points of the constellation with equal probability. STBC based STTC site diversity outperforms B-STTC site diversity as it gains additional diversity gain. It clearly highlights that the STBC based STTC site diversity is provides better performance and is best opted for the system.

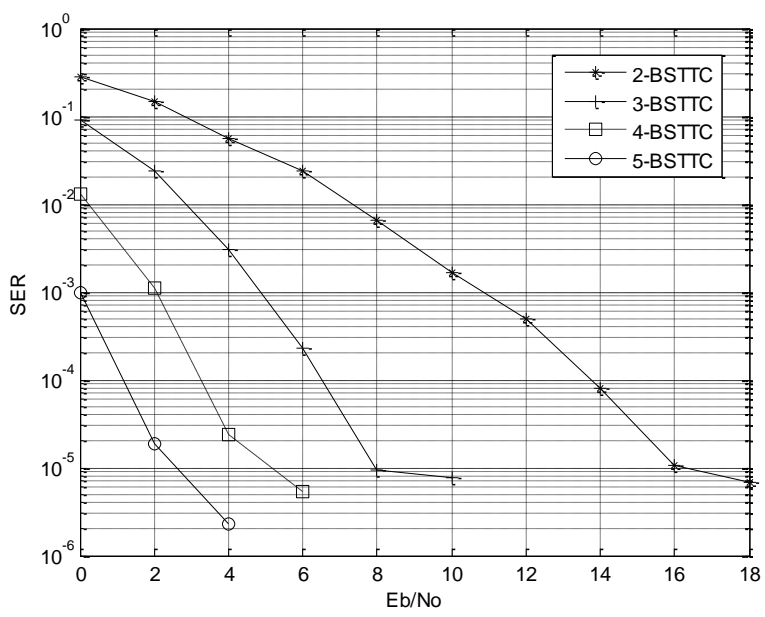

Figure 7. Performance of the system with B-STTC site diversity for various antennas

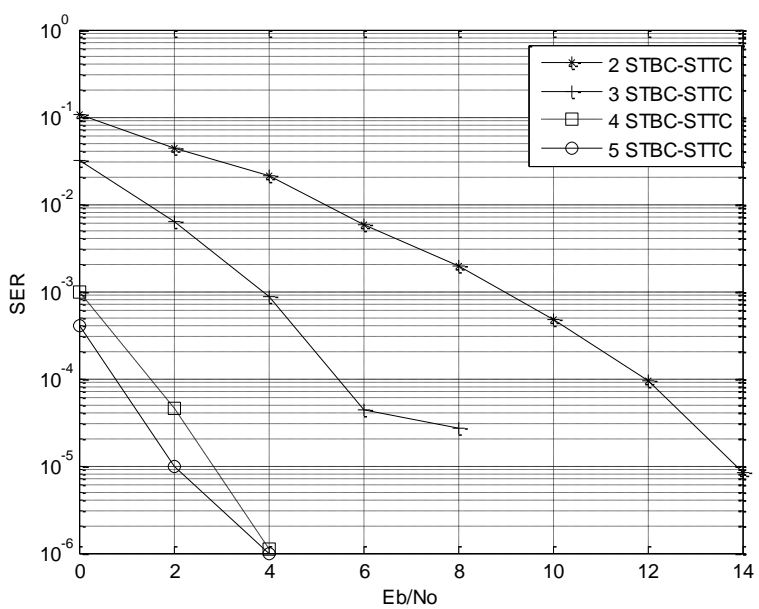

Figure 8. Performance of the system with STBC based STTC site diversity for various antennas

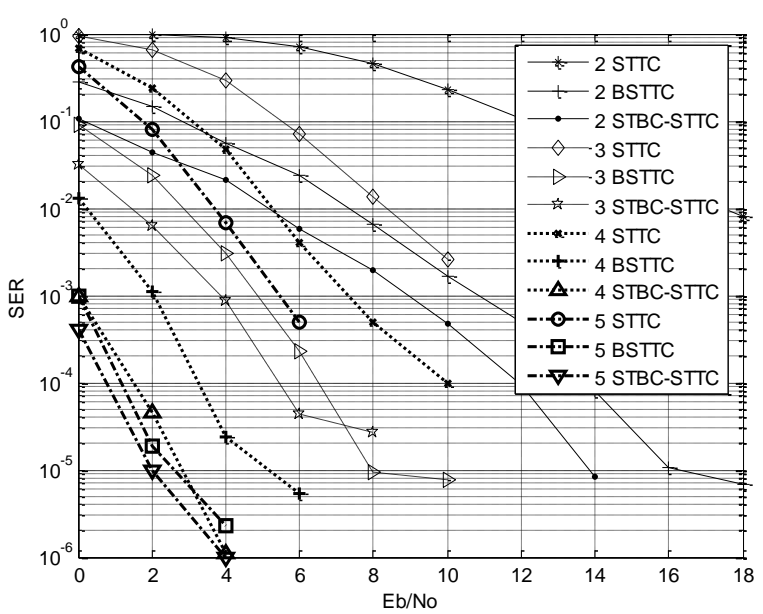


Figure 9. Performance comparison of the system with STTC, B-STTC and STBC based STTC site diversity for various transmit and receive antennas based on SU(3)", IEEE Transactions on Signal Processing, vol. 53, no. 10 , pp.3688-3702, Oct. 2005.

\section{CONCLUSION}

In this paper, site diversity scheme for MC-CDMA system is proposed using B-STTC and STBC based STTC with MIMO technique to improve the performance of mobile terminals in the downlink. These methods considerably minimise multi-cell interference by jointly consuming the diversity gain and coding gain. Coding gain was further improved in B-STTC as they use points of constellation with equal probability. With STBC based STTC site diversity technique, the performance of MC-CDMA system achieves better reduction in error rates with additional diversity gain. Simulation results shows that STBC based STTC site diversity outperforms B-STTC based site diversity in terms of SER and is best opted for MC-CDMA system.

\section{REFERENCES}

[1] S. Hara and R. Prasad, "Design and performance of multicarrier CDMA system in frequency selective Rayleigh fading channels", IEEE Transactions on Vehicular Technology, vol. 48, no.5, pp.1584-1595, Sep. 1999.

[2] S. Hara and R. Prasad, "Overview of multi carrier CDMA", IEEE Communication Magazine, vol. 35, no.12, pp. 126- 133, Dec. 1997.

[3] S. Chatterjee, W.A.C. Fernando and M.K. Wasantha, "Adaptive modulation based MC-CDMA systems for $4 \mathrm{G}$ wireless consumer applications", IEEE Transactions on Consumer Electronics, vol.49, no.4, pp.995-1003, Nov. 2003.

[4] G. Foschini and M. Gans, "On the limits of wireless communications in a fading environment when using multiple antenna", Wireless Personal Commuication, vol. 6, no. 3, pp. 311-335, Mar. 1998.

[5] S.M. Alamouti, "A simple transmit diversity technique for wireless communications", IEEE Journal on Selected Areas in Communication, vol.16, no.8, pp. 1451-1458, Oct. 1998.

[6] M. Inoue, T. Fujii and M. Nakagawa, "Space time transmit site diversity for OFDM multi- base station system", Proceedings of $4^{\text {th }}$ International Workshop on Mobile and Wireless Communications Network, Sweden, pp. 30-34, Sep. 2002.

[7] D. Wong and T.J Lim, "Soft handoffs in CDMA mobile systems", IEEE Personal Communications, vol.36, no.6, pp.6-17, Dec.1997.

[8] N. Kumarathran, M.Padmavathy and P.Dananjayan, "Performance improvement of MC-CDMA system through STBC site diversity", International Journal of Emerging Technologies and Applications in Engineering, Technologies and Sciences, Dec. 2008, ISBN : 0974-3588.

[9] V. Tarokh, N. Seshadri and A.R. Calderbank, "Space-time codes for high data rate wireless communication: Performance criterion and code construction", IEEE Transactions on Information Theory, vol. 44, no.2, pp.744-765, March 1998.

[10] N. Kumarathran, S.Jayapriya and P.Dananjayan, "Performance improvement of MC-CDMA system through STTC site diversity", Proceedings of International Conference on Computing, Communication and Networking, ICCCN-08, India, Dec. 2008.

[11] T.M.H. Ngo, G. Zaharia, S. Bougeard and J. F. Helard, "Design of balanced QPSK space-time trellis codes for several transmit antennas", Proceedings of International Symposium on Signals, Circuits and Systems, Lasi, vol.2, pp. 1-4, Jul. 2007.

[12] G. Ferre, J. P. Cances , V. Meghdadi and J. M. Dumas, "STBC based STTC codes optimized designs for three transmit antennas", Special issue on Communication Systems, Networks And Digital Signal Processing, vol. 3, no.3, pp.110-118, July 2007.

[13] A.F. Naguib, V.Tarokh , N. Seshadri and A.R. Calderbank, "A space-time coding modem for high-data-rate wireless communications", IEEE Journal on Selected Areas in communications, vol.16, no.8, pp.1459-1478, Oct. 1998.

[14] Z. Chen, J. Yuan and B. Vucetic, “ Improved space-time trellis coded modulation scheme on slow Rayleigh fading channels", IEEE Electronics Letters, vol.37, no.7, pp.440-451, Mar. 2001

[15] Y. Jing and B. Hassibi, "Three transmit antenna space-time codes

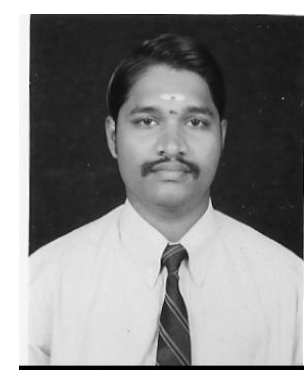

N.Kumaratharan received his B.E degree in Electrical and Electronics Engineering from University of Madras in 2001. He received his Masters Degree in Applied Electronics from Anna University, Guindy, Chennai in 2004. He has two and half years of teaching experience. $\mathrm{He}$ is presently pursuing his research in the area of wireless communication. He has published two papers in International Journal and three papers in International Conference. His area of interest includes Broadband and mobile ccommunication.

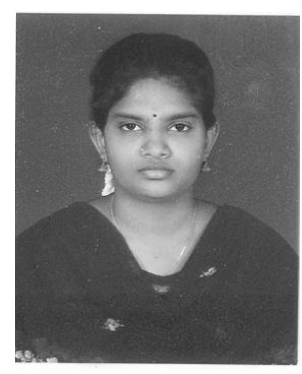

S. Jayapriya received her B. Tech degree in Electronics and communication engineering from Sri Manakula vinayagar engineering college affiliated to Pondicherry University in 2007. She is pursuing her M.Tech in Pondicherry engineering college, Pondicherry. Her area of interests include Digital and wireless communication.

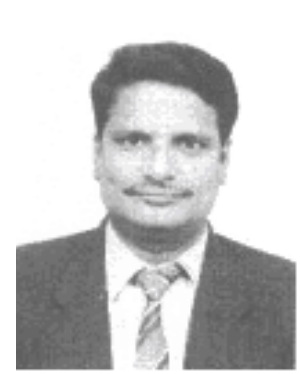

P. Dananjayan received Bachelor of Science from University of Madras in 1979, Bachelor of Technology in 1982 and Master of Engineering in 1984 from the Madras Institute of Technology, Chennai and Ph.D. degree from Anna University, Chennai in 1998. He is working as a Professor and Head of the Department of Electronics and Communication Engineering, Pondicherry Engineering College, Pondicherry, India. He has more than 60 publications in National and International Journals. He has presented more than 130 papers in National and International conferences. He has guided $6 \mathrm{Ph} . \mathrm{D}$ candidates and is currently guiding eight Ph.D students. His areas of interest include Spread spectrum techniques, Wireless communication and Sensor networks. 